\title{
Fault Detection Enhancement in Rolling Element Bearings via Peak-Based Multiscale Decomposition and Envelope Demodulation
}

\author{
Hua-Qing Wang, ${ }^{1}$ Wei Hou, ${ }^{1}$ Gang Tang, ${ }^{1}$ Hong-Fang Yuan, ${ }^{2}$ \\ Qing-Liang Zhao, ${ }^{1}$ and Xi Cao ${ }^{2}$ \\ ${ }^{1}$ School of Mechanical and Electrical Engineering, Beijing University of Chemical Technology, Beijing 100029, China \\ ${ }^{2}$ School of Information Science and Technology, Beijing University of Chemical Technology, Beijing 100029, China
}

Correspondence should be addressed to Gang Tang; tanggang@mail.buct.edu.cn

Received 31 March 2014; Accepted 7 May 2014; Published 27 May 2014

Academic Editor: Ruqiang Yan

Copyright (C) 2014 Hua-Qing Wang et al. This is an open access article distributed under the Creative Commons Attribution License, which permits unrestricted use, distribution, and reproduction in any medium, provided the original work is properly cited.

\begin{abstract}
Vibration signals of rolling element bearings faults are usually immersed in background noise, which makes it difficult to detect the faults. Wavelet-based methods being used commonly can reduce some types of noise, but there is still plenty of room for improvement due to the insufficient sparseness of vibration signals in wavelet domain. In this work, in order to eliminate noise and enhance the weak fault detection, a new kind of peak-based approach combined with multiscale decomposition and envelope demodulation is developed. First, to preserve effective middle-low frequency signals while making high frequency noise more significant, a peak-based piecewise recombination is utilized to convert middle frequency components into low frequency ones. The newly generated signal becomes so smoother that it will have a sparser representation in wavelet domain. Then a noise threshold is applied after wavelet multiscale decomposition, followed by inverse wavelet transform and backward peak-based piecewise transform. Finally, the amplitude of fault characteristic frequency is enhanced by means of envelope demodulation. The effectiveness of the proposed method is validated by rolling bearings faults experiments. Compared with traditional wavelet-based analysis, experimental results show that fault features can be enhanced significantly and detected easily by the proposed method.
\end{abstract}

\section{Introduction}

A rolling bearing is one of the most widely used elements in rotating machinery. As a critical component, it carries most of the load during the running of rotating machinery. If rolling bearing fails, serious problems arise, which will in turn result in the decrease of production efficiency and large economic loss. Records show that faulty bearings contribute to about thirty percent of failures in rotating machinery. Thus, it is of great importance to study the effective fault diagnosis approaches for rolling bearings.

Various methods have been developed for bearing fault diagnosis and condition monitoring, such as vibration monitoring, temperature monitoring, chemical analysis, acoustic emission monitoring, sound pressure monitoring, and laser monitoring [1]. Vibration signal analysis is one of the most efficient methods thanks to the useful information of machine's work status carried by vibration signals [2-4]. To extract fault information, for example, partial defects of bearings, vibration signals are usually processed in time domain, frequency domain, or both $[5,6]$. In time domain, some statistical parameters, for example, probability density, kurtosis, root mean square, or skewness, have been introduced into bearing defect detection [7]. However, these fault indicators are not effective in all cases, especially for weak fault signals. Therefore, analysis approaches in frequency domain are developed to detect bearing defect fault. Based on the efficiency of modern fast Fourier transform (FFT), spectral analysis of vibration signals is widely used to extract characteristic defect frequencies. In fact, bearing fault signal is 
amplitude modulated at its characteristic defect frequencies, so a preprocessing of demodulation should be performed before FFT applied. Traditionally, envelope demodulation is used to detect the fault frequency $[5,8]$. For obvious fault features, traditional envelope demodulation has notable advantages in fault type recognition. However, it does not work well in many cases for the detection of weak fault features. To solve this problem, various approaches have been developed to denoise vibration signals and to enhance the characteristic defect frequency. Cyclostationary analysis [9], for example, spectral autocorrelation analysis [10], is such a way. In recent years, some methods based on time-frequency analysis, such as empirical mode decomposition (EMD) [1113], local mean decomposition (LMD) [14, 15], and wavelet transform $[16,17]$, are also developed to enhance fault features and detect failures. Both EMD and LMD are sensitive to noise level, but they are so hard to implement properly since these algorithms contain imperfections. In addition to the mentioned signal processing approaches, some other strategies are also introduced for fault features extraction as well as pattern recognition, for example, symptom parameter waves [18] and fuzzy diagnosis method [19].

As mentioned above, signal's decomposition or sparse representation is the basis of those methods. However, because of the existing high frequency components, vibration signals are not always sparse enough after decomposition. As a popular strategy for bearing vibration signals' processing, wavelet-based analysis is effective for feature extraction from smooth signals, signal denoising, and fault feature enhancement [20-22]. But it cannot always represent vibration signals sparsely enough, especially for those mixed by plenty of interferential signals generated by other related rolling elements. This will be illustrated further with a typical example in the next section. In related research areas, a so-called peak transform based on piecewise curve recombination has been developed. The idea is to adjust piecewise curves to improve the smoothness of the target signals or images, and it has been used in 2D image representation and coding $[23,24]$. To our knowledge, there is no report in the literature by far on its applications to bearing vibration signal analysis.

In order to overcome those disadvantages of waveletbased methods for weak signals processing of bearing faults, this paper developed a new multiscale decomposition strategy with sparsity-promoting by recombination of piecewise signals under different frequencies. The new strategy improves the sparsity of original vibration signals and is of great significance in fault signals denoising and enhancement. Then the wavelet coefficients of vibration signals will be sparse enough, and a threshold method based on wavelet decomposition can eliminate most noise. Finally, the FFT-based Hilbert transform is conducted for signal demodulation and extracting fault signature of characteristic defect frequency. Fault features will be significantly enhanced and easily detected through the proposed method.

The paper is organized as follows. Section 2 illustrates the sparseness of vibration signals in wavelet domain, which is also the motivation of this work. The proposed method of peak-based multiscale decomposition and envelope demodulation is introduced in Section 3. Some experimental results

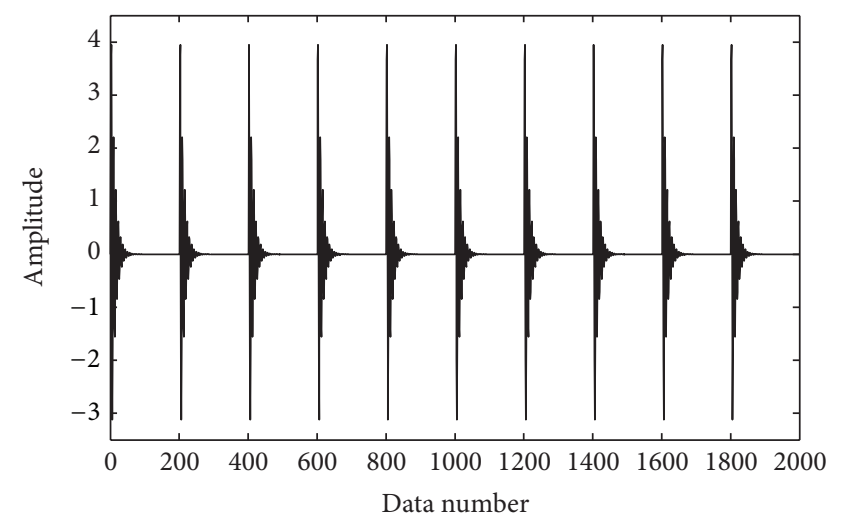

FIGURE 1: Vibration waveform of a bearing with a point defect on the outer race.

to show the effectiveness of the method are presented in Section 4. Finally, discussions and conclusions are presented in Section 5.

\section{Vibration Signal of Rolling Bearing Faults and Its Sparsity in Wavelet Domain}

Vibration signal is always acquired by sensors related to acceleration, velocity, or displacement measurement. A series of signal processing steps are then applied to make the fault features easy to be distinguished. Basically, the key of vibration signal analysis is to find out appropriate parameters describing the running conditions of bearings clearly. The commonly used characteristic defect frequency is one of the most effective indicators for rolling bearing's fault. If local defects exist in a bearing, the measured vibration signal will be amplitude modulated. Its carrier wave is a kind of the bearing's inherent vibration with high frequency, while its modulating wave is a pass vibration corresponding to the local defects, whose frequency is called ball pass frequency or characteristic defect frequency. The latter is usually utilized to determine whether a fault exists, and it is also adopted by this paper.

In order to reduce the potential impact of signal attenuation through complex paths, sensors are often placed as close to the bearings as possible, for example, bearing housing. However, transmission path still exists between the impact point and the vibration measurement point, which would make signals more complex and hard to be demodulated. If defects exist, a fault in one surface of bearings would strike another; then a force impulse will be generated and flowing resonances in the bearing and machine will also be excited [25]. A series of impulse responses will be generated and last continuously, which may be amplitude modulated caused by the fault passing through the load zone or the mentioned varying transmission paths. Therefore, the ideal bearing defect signal is a kind of periodical impulse-like signal with high frequency nonsmooth components. A typical vibration signal of a bearing is shown in Figure 1; the vibration waveform is obviously impacted by an existing point defect on the outer race. In practice, in addition to bearing condition 


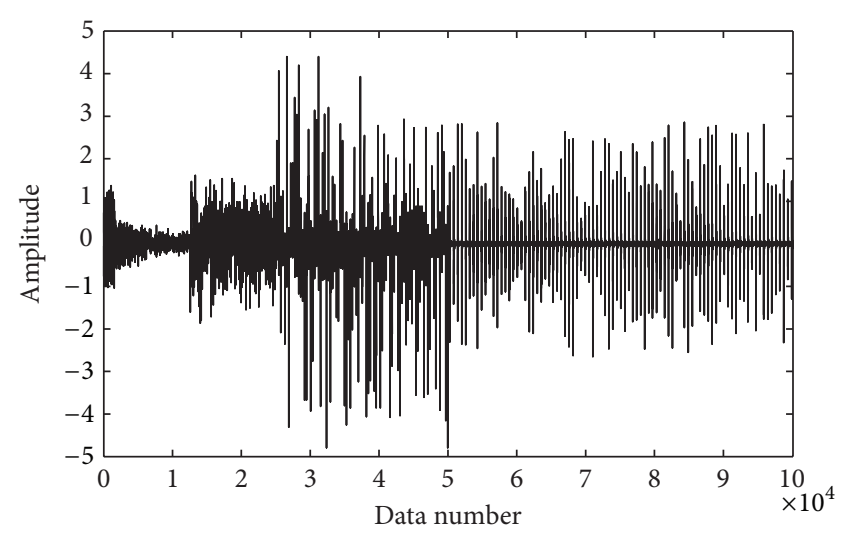

FIGURE 2: Wavelet coefficients of the bearing fault signal in Figure 1.

information, the measured vibration signals are often mixed with lots of interfering information from other components. Meanwhile, various types of noise also make the signal more complicated. In addition to the noise introduced by measurement instruments, interfering signals can also be generated accompanied with the existence of transmission path between local impact point and vibration measurement point. In a word, bearing vibration signal is nonstationary with wide frequency band, which makes the bearing fault signature extremely weak to be detected.

Existing wavelet-based denoising methods mainly rely on the sparseness of wavelet coefficients [17]. If a signal is not sparse enough in wavelet domain, it becomes challenging for feature extraction by wavelet analysis due to the difficulties to distinguish required features from large number of wavelet coefficients. Common vibration signals from faulty bearings are often composed of impulse components. A typical vibration signal is shown in Figure 1, and its wavelet coefficients are shown in Figure 2, from which we can see that most coefficients are nonzero, that is, not sparse enough. Experiments show that this is applicable to fault vibration signals of inner race, outer race, and ball element. Thus it is important to find out a proper way to improve the sparseness, as well as reducing noise and enhancing the weak fault features.

\section{The Proposed Method}

3.1. The Basic Principle of Peak-Based Piecewise Recombination. Peak-based piecewise recombination (PPR) or socalled peak transform is a kind of nonlinear geometric transform to make the signal smoother through transforming high frequency subsections to low frequency ones. Here, a brief description of PPR is given as follows; for more information please refer to [23].

For a continuous function $f(x)$ that has $N$-point peaks (or break points), $x_{i}(i=1 \cdots N)$ is defined over $[a, b]$. In fact, $f(x)$ can be viewed as a piecewise function with $N+1$ combined curves, where the $N$ peaks' positions are the connection points. Then the $i$ th piecewise curve can be called subfunction $f_{i}(x)$ over interval $[a, b]$. The $N$-point forward
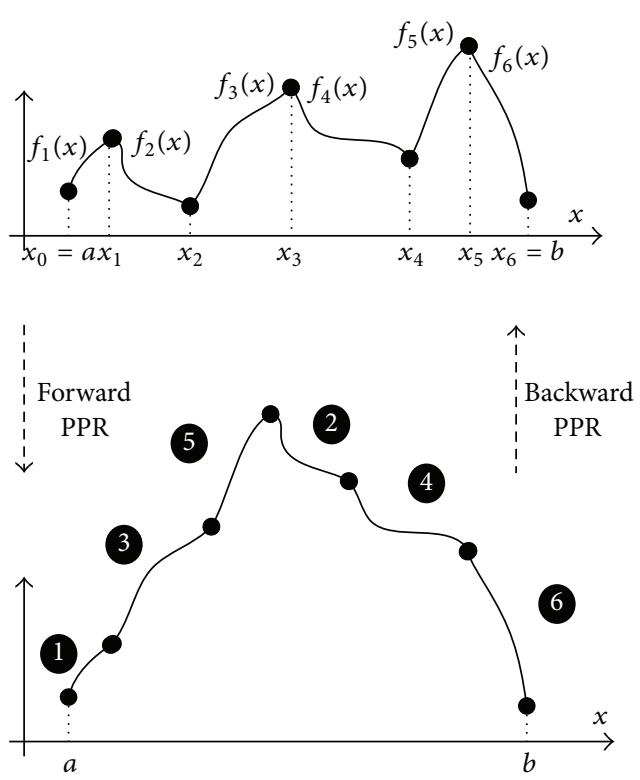

FIGURE 3: Schematic diagram of five-point peak-based piecewise recombination.

transform of peak-based piecewise recombination can be defined as

$$
P F[f(x)]=g_{o}(x) \oplus g_{e}(x),
$$

where

$$
\begin{aligned}
& g_{o}(x)=f_{1}(x) \oplus f_{3}(x) \oplus \cdots \oplus f_{2\lfloor(N-1) / 2\rfloor+1}(x), \\
& g_{e}(x)=f_{2}(x) \oplus f_{4}(x) \oplus \cdots \oplus f_{2\lfloor N / 2\rfloor}(x)
\end{aligned}
$$

are the cascades of all odd- and even-numbered curve segments, respectively. Here, $2\lfloor(N-1) / 2\rfloor+1$ and $2\lfloor N / 2\rfloor$ are, respectively, the largest odd and even integers that are less than or equal to $N$.

Geometrically speaking, as illustrated in Figure 3, all piecewise curves with similar positive slopes are grouped together and cascaded to be a new curve, while all negative ones are grouped into another new curve; finally, these two new subcurves are recombined to be a new curve. It is apparent that the recombination only changes the order of the piecewise curves and is reversible. The backward transform can be done by simply recascading them according to their original orders, which is symbolized as $P F^{-1}[f(x)]$ according to (1).

It should be noted that, although the forward and backward transforms discussed above are defined for curve functions, they can also be defined in similar manners for discretetime signals of rolling bearings' vibration.

3.2. Wavelet Decomposition. Wavelet $\psi_{(a, b)}(t)$ is obtained by translation and dilation based on a defined single function $\psi(t)$ as

$$
\psi_{(a, b)}(t)=\frac{1}{\sqrt{a}} \psi\left(\frac{t-b}{a}\right),
$$


where $a>0$ is the so-called scaling parameter and $b \in R$ is the parameter denoting time localization, which can be continuous or discrete. $\psi(t)$ is called "mother wavelet" to generate wavelets $\psi_{(a, b)}(t)$.

Given a signal $x(t)$ with finite energy, the wavelet transform with analytic wavelet $\psi(t)$ can be viewed as the convolution of $x(t)$ with a scaled and conjugated wavelet

$$
W(a, b)=\frac{1}{\sqrt{a}} \int_{-\infty}^{\infty} x(t) \psi^{*}\left(\frac{t-b}{a}\right) d t,
$$

where $\psi^{*}(t)$ stands for the complex conjugation of $\psi(t)$.

The wavelet transform $W(a, b)$ can be considered as a function of translation $b$ at each scale $a$. From (4), we can see that wavelet transform is a kind of time-frequency analysis or a time-scaled analysis. Different from analysis either in time domain or in frequency domain, or even different from that in time-frequency domain but with fixed-length windows by short time Fourier transform (SFFT), vibration signals can be decomposed by wavelet transform with multiscale analysis through dilation and translation, so that the time-frequency features of vibration signals can be more effectively extracted.

Wavelet transform is also reversible as follows:

$$
x(t)=C_{\psi}^{-1} \iint W(a, b) \psi_{(a, b)}(t) \frac{d a}{a^{2}} d b,
$$

where

$$
\begin{aligned}
& C_{\psi}=\int_{-\infty}^{\infty} \frac{|\widehat{\psi(\omega)}|^{2}}{|\omega|} d \omega<\infty, \\
& \widehat{\psi}(w)=\int \psi(t) \exp (-j w t) d t
\end{aligned}
$$

which provides the possibility to reconstruct the original vibration signals.

3.3. Envelope Demodulation. As mentioned in the previous sections, if bearing defects exist, the measured vibration signal would be amplitude modulated at its characteristic defect frequency. The modulating wave is a pass vibration signal corresponding to local defects, and various demodulation techniques have been developed for the separation. In this work, an envelope demodulation method based on Hilbert transform is employed.

For a continuous time signal $x(t)$, the Hilbert transform $\widehat{x(t)}$ is defined as

$$
\widehat{x(t)}=\frac{1}{\pi} \int_{-\infty}^{+\infty} \frac{x(\tau)}{(t-\tau)} d \tau
$$

Then, combine $x(t)$ and $\widehat{x(t)}$ to form a new analytic signal

$$
g(t)=x(t)+j \widehat{x(t)} .
$$

The envelope of $x(t)$ is defined as the amplitude of $g(t)$ :

$$
A(t)=\sqrt{[x(t)]^{2}+[x(t)]^{2}} .
$$

After Hilbert transform, envelope spectra could be obtained by FFT to extract characteristic defect frequencies.
3.4. Enhancement Frame with Peak-Based Multiscale Decomposition and Envelope Demodulation. Wavelet transform is a kind of time-frequency analysis method for signal denoising, by adjusting the discrete detail coefficients and approximation coefficients obtained from multilevel decomposition. This scheme is called threshold processing, one of whose premises is the sparse representation of signals in wavelet domain. However, different from that of perfect bearing, the vibration signal energy of bearings with defects increases at high frequencies. Thus, vibration signals are not always sparse in wavelet domain. Piecewise curve recombination can convert energies at high frequencies to ones at low frequencies. In this way, signal energies are focused in low frequency subbands, so that the piecewise recombination can improve signal's smoothness as well as its sparseness in wavelet domain.

The present paper proposed an enhancement frame for vibration signals by the combination of peak-based multiscale decomposition and envelope demodulation. The procedure is described as follows.

(1) Peak-based piecewise recombination of vibration signal: let $x(t)$ denote the measured vibration signal, firstly; then, transform the original signal $x(t)$ to smooth signal $P F[f(x)]$ through piecewise recombination according to Equation (1). The key is to determine the peaks and to recombine different segments. In the present work, a cascading strategy related to local minimum and maximum is employed. The endpoint of a segment is chosen as a peak. If two adjacent peaks are equal, one of them is defined as the peak.

(2) Wavelet decomposition of the new recombined signal: choose a wavelet basis (e.g., Morlet wavelet or Db4 wavelet); then decompose the new signal $P F[f(x)]$ at level $N$ :

$$
W(a, b)=\frac{1}{\sqrt{a}} \int_{-\infty}^{\infty} P F[x(t)] \psi^{*}\left(\frac{t-b}{a}\right) d t .
$$

(3) Coefficients threshold: set a threshold value and apply it to the approximation coefficients at level $N$ and the detail coefficients at levels 1 to $N$. The hard threshold can be expressed as

$$
y_{s}= \begin{cases}y & |y|>t \\ 0 & |y|<t\end{cases}
$$

where $t$ is the selected threshold, $y$ is the original wavelet coefficients, and $y_{s}$ is the wavelet coefficients after thresholding. In this work, the value which covers about $95 \%$ of the smallest wavelet coefficients is set as the threshold value. Then $5 \%$ of the largest wavelet coefficients are kept as nonzero ones to reserve the signal feature.

(4) Signal recovery: reconstruct the vibration signal based on the modified approximation coefficients at level $N$ and the modified detail coefficients at levels 1 to $N$ :

$$
P F^{\prime}[x(t)]=C_{\psi}^{-1} \iint W^{\prime}(a, b) \psi_{(a, b)}(t) \frac{d a}{a^{2}} d b .
$$




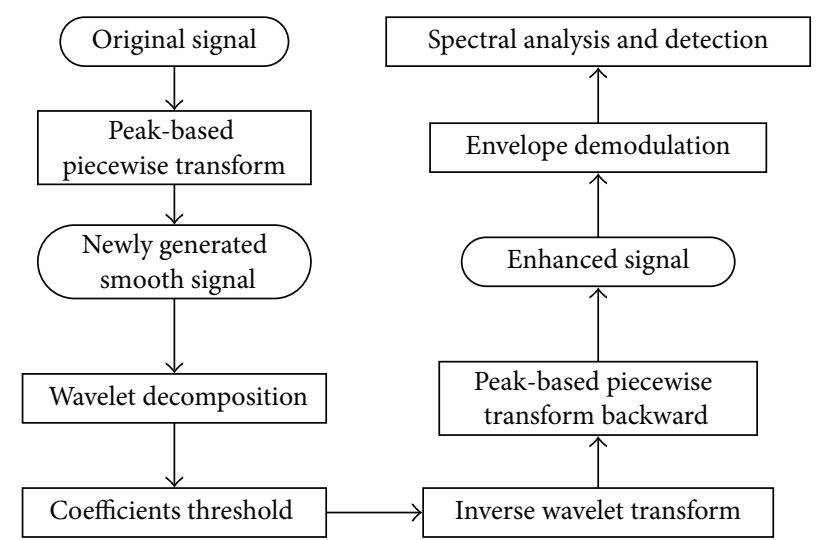

FIGURE 4: Flowchart of the proposed enhancement scheme for weak fault signal.

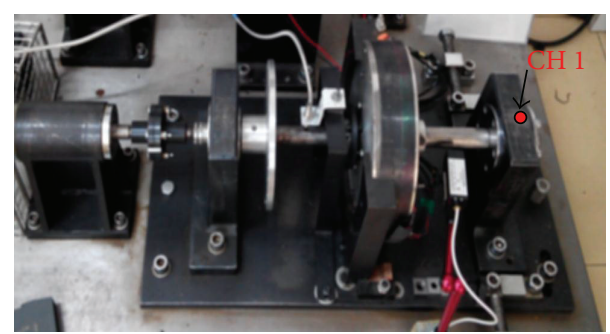

FIGURE 5: Fault rig of a roller element bearing.

(5) Piecewise signal backward transform: put the processed signal segments back to their original positions; then a new denoised vibration signal $x^{\prime}(t)$ is generated.

(6) Envelope demodulation: according to (9), use Hilbert transform to obtain the envelope $A(t)$ of signal $x^{\prime}(t)$.

(7) Extraction of characteristic defect frequency: get the envelope spectra via Fourier analysis and distinguish different fault types of rolling bearings.

In summary, these steps are shown as in Figure 4.

\section{Experimental Results and Discussions}

4.1. Experiment Setup. To verify the effectiveness of the proposed method, experiments are designed for fault rigs of roller element bearing, as shown in Figure 5. The rig is composed of a motor, a coupling, a rotor, and a shaft with two roller bearings. Basically, the sensors are located at the position near the bearings to mitigate the effects of signal attenuation. Usually, bearing housing is one of the best locations for bearing arrangement. Therefore, here, an accelerometer is located on the bearing housing to acquire the vibration signals.

In the experiments, a single point defect is introduced in inner raceway, outer raceway, and ball element of different bearings, using electron-discharge machining with fault widths of $3 \mathrm{~mm}, 7 \mathrm{~mm}$, and $7 \mathrm{~mm}$ and depths of $5 \mathrm{~mm}$, $25 \mathrm{~mm}$, and $25 \mathrm{~mm}$, respectively.
Vibration signals are measured by an accelerometer, located at the top of the bearing house $(\mathrm{CH} 1$, as shown in Figure 5). In all experiments, the sample frequency is $100 \mathrm{kHz}$ and the shaft speed is finite, $1300 \mathrm{rpm}$. Four common conditions are studied in the present paper, including a perfect healthy bearing and three other bearings with point defect on the outer race, on the inner race bearing, and on the ball element.

In theory, elements of roller bearings have their own specific rotational frequencies, which may appear in envelope spectra if defects exist. These defect frequencies are called ball pass frequencies. For a bearing with a stationary outer race, these frequencies can be given by the following formulas:

$$
\begin{aligned}
& \text { outer race defect frequency, } w_{\mathrm{od}}=\frac{Z w_{s}}{2 d}\left(1-\frac{d}{D} \cos \alpha\right), \\
& \text { inner race defect frequency, } w_{\mathrm{id}}=\frac{Z w_{s}}{2}\left(1+\frac{d}{D} \cos \alpha\right), \\
& \text { rolling element defect frequency, } w_{\mathrm{re}}=w_{s} \frac{D}{2 d} \\
& \times\left(1-\frac{d^{2}}{D^{2}} \cos ^{2} \alpha\right),
\end{aligned}
$$

where $w_{s}$ is the shaft rotation frequency in $\mathrm{rad} / \mathrm{s}, d$ is the diameter of rolling element, $D$ is the pitch diameter, $Z$ is the number of rolling elements, and $\alpha$ is the contact angle.

In the present experiments, outer race defect frequency, inner race defect frequency, and rolling element defect frequency are $86.32 \mathrm{~Hz}, 145.84 \mathrm{~Hz}$, and $51.13 \mathrm{~Hz}$, respectively.

4.2. Vibration Signals of Typical Bearing Faults and Their Envelop Spectra. The time-domain waveforms of bearing vibration signals obtained from accelerometers are shown in Figure 6, from which we can see that, different from that of perfect bearings as shown in Figure 6(a), obvious impulsive phenomenon exists in faulty vibration signals of fault bearings as shown in Figures 6(b), 6(c), and 6(d).

To investigate the differences of the signals and to distinguish different fault types, envelope demodulation is applied first to vibration signals of the three typical bearing faults, that is, outer race fault, inner race fault, and roller element fault. The results are shown in Figures 7, 8, and 9. In Figure 7, the frequency magnitude of outer race defect is lower than the second harmonic frequency of outer race defect. In Figure 8, although the inner race defect frequency slightly sticks out, it is still possibly confused by the sidelobes with almost similar magnitudes. In Figure 9, it is almost impossible to figure out the calculated ball pass frequency of $51.13 \mathrm{~Hz}$. From the figures, we can see that, simply based on envelope demodulation, it is difficult to identify the fault frequencies from those of other components, especially for weak fault signals.

4.3. Wavelet Coefficients Distributions of the Vibration Signals with and without Peak-Based Piecewise Recombination. Before envelope demodulation, wavelet decomposition is 


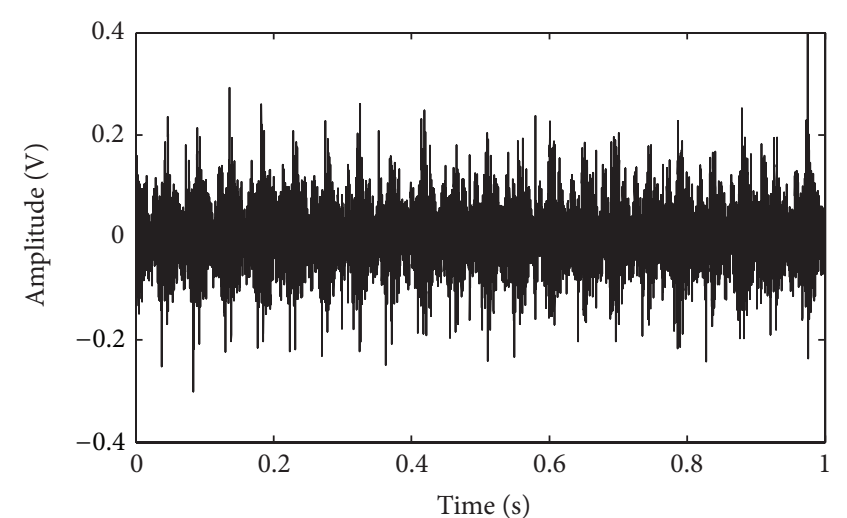

(a)

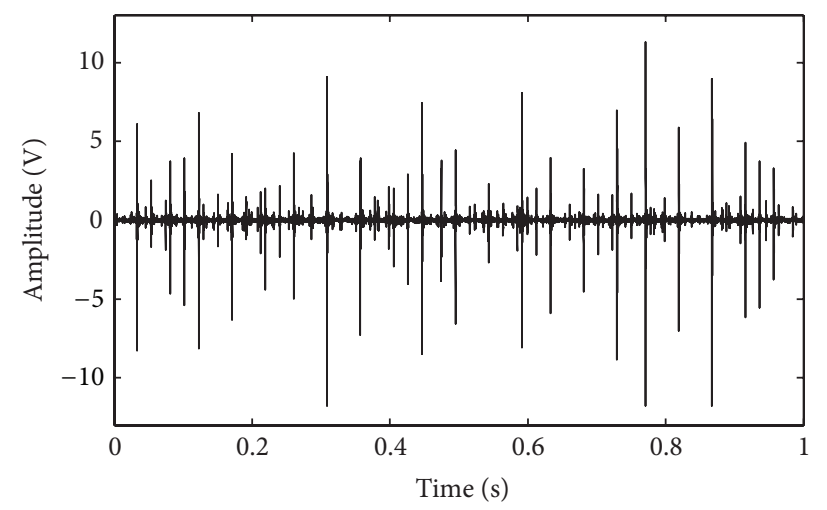

(c)

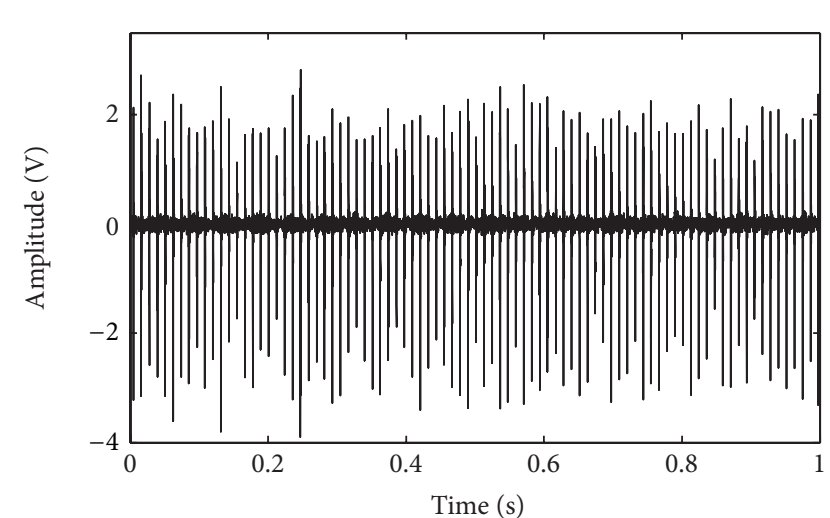

(b)

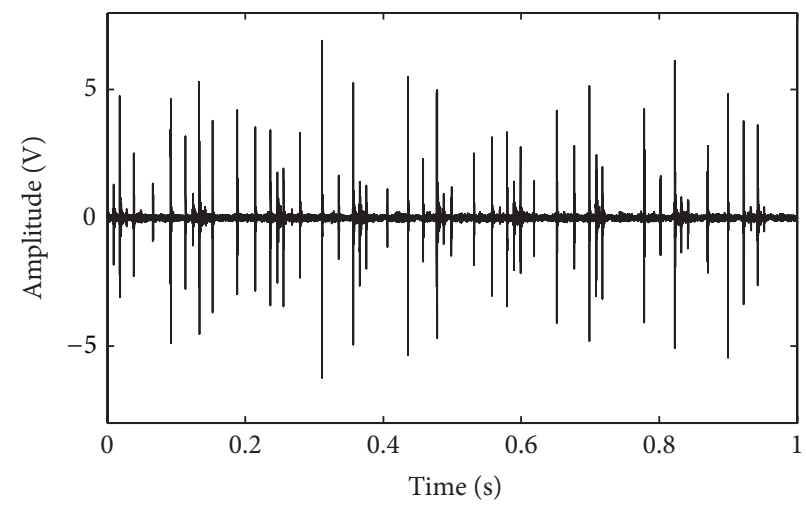

(d)

FIGURE 6: Vibration signals of rolling bearings: (a) a normal bearing and bearings with a point defect on (b) outer race, (c) inner race bearing, and (d) ball element.

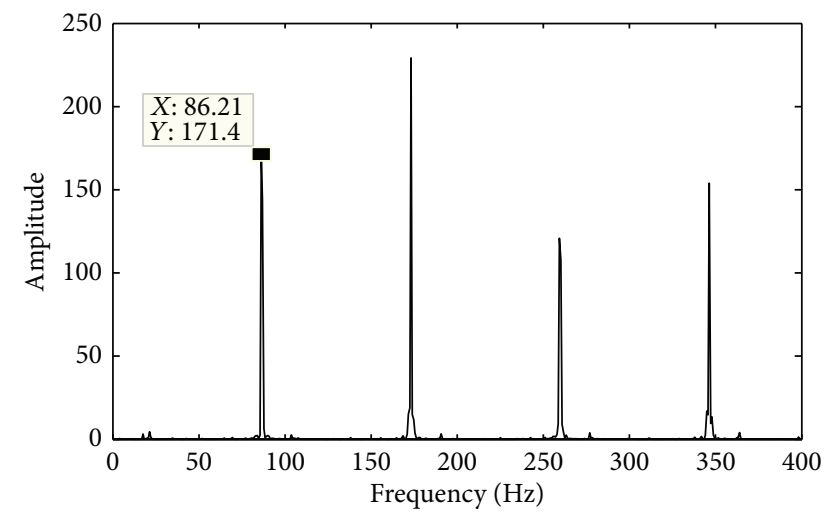

FIGURE 7: Envelope spectra of vibration signal in Figure 6(b) induced by outer race fault.

always applied to denoise and enhance the weak fault signals. However, vibration signals are not always sparse enough in wavelet domain, which would affect the effectiveness of the enhancement. As mentioned above, peak-based piecewise recombination can help to improve the smoothness of vibration signals and make their energies more concentrated in

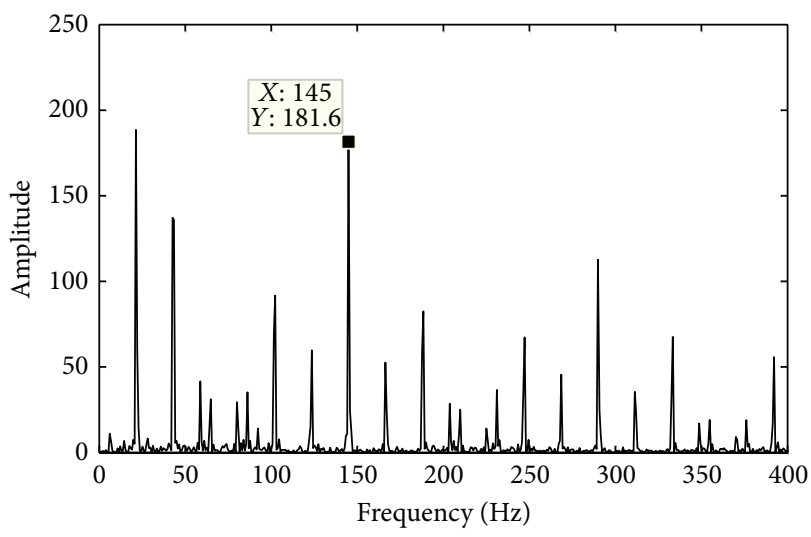

Figure 8: Envelope spectra of vibration signal in Figure 6(c) induced by inner race fault.

wavelet domain. Comparisons between wavelet decompositions with and without peak-based piecewise recombination are illustrated in this section.

Taking outer race fault as an example, the time-domain vibration signal of Figure 6(b) is converted to that of Figure 10 after applying piecewise recombination. The signal becomes much smoother than the original signal after piecewise 


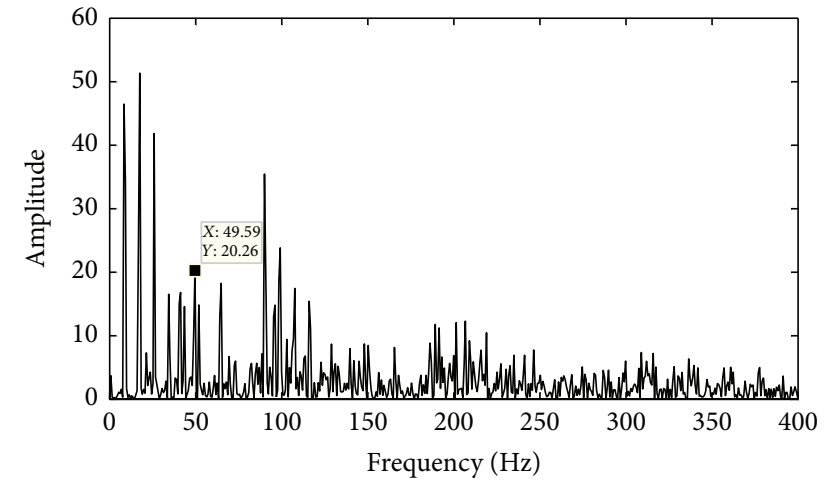

FIGURE 9: Envelope spectra of vibration signal in Figure 6(d) induced by roller element fault.

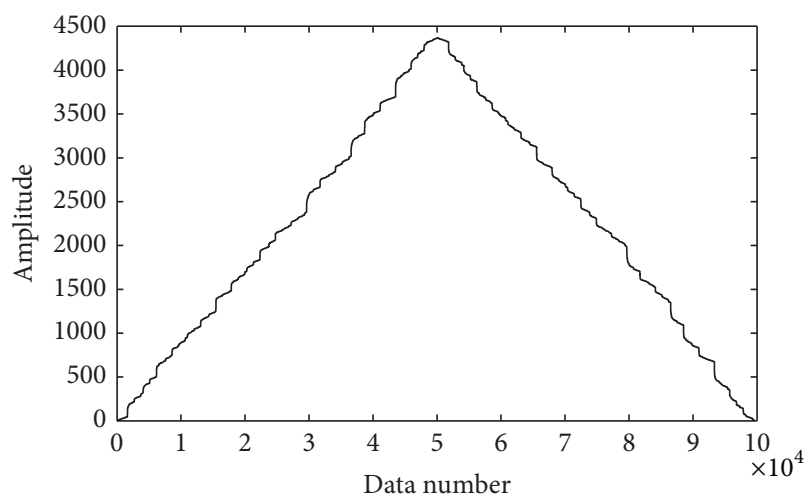

FIgURE 10: Vibration signal induced by outer race fault in Figure 6(b) after peak-based piecewise recombination.

TABLE 1: Energy distributions of the outer race fault signal's wavelet coefficients.

\begin{tabular}{lcc}
\hline Frequency band & Low frequency & High frequency \\
\hline Energies without PPR & $49.31 \%$ & $50.69 \%$ \\
Energies with PPR & $99.9 \%$ & $0.1 \%$ \\
\hline
\end{tabular}

recombination; that is, high frequency components have been converted to low frequency ones.

For comparisons, then wavelet transform is applied to signals in Figures 6(b) and 10, respectively, where 6-level decomposition with $\mathrm{db} 4$ wavelet basis is employed. Both distributions of the wavelet coefficients are shown in Figure 11. Without piecewise recombination, most wavelet coefficients are nonzero, that is, not sparse enough, as depicted in Figure 11(a). However, it is improved significantly with preprocessing of peak-based piecewise recombination, as depicted in Figure 11(b). Energies of the signal in wavelet domain are concentrated in only a few low frequency intervals, and almost all detail coefficients at scales 1 to 6 are close to zero. It implies that peak-based piecewise recombination can significantly improve the sparseness of vibration signals in wavelet domain.

Statistically, energy distributions of wavelet coefficients obtained by the two methods are listed in Table 1, from

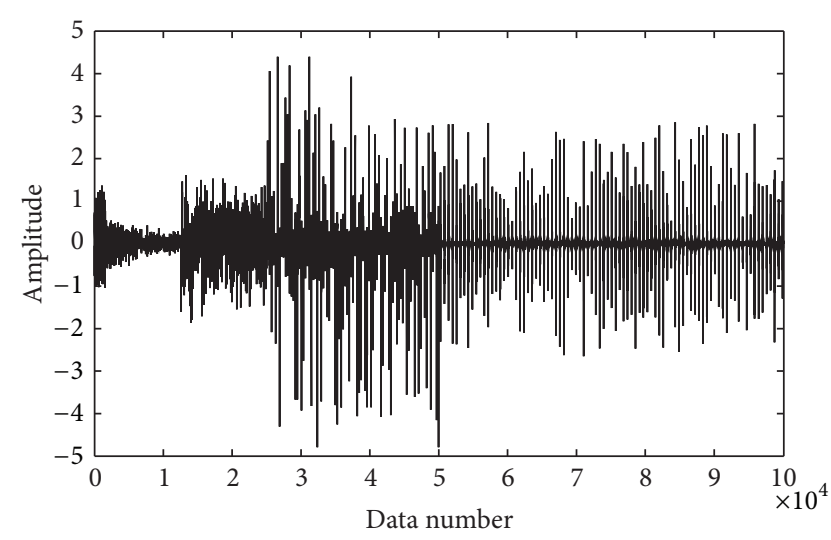

(a)

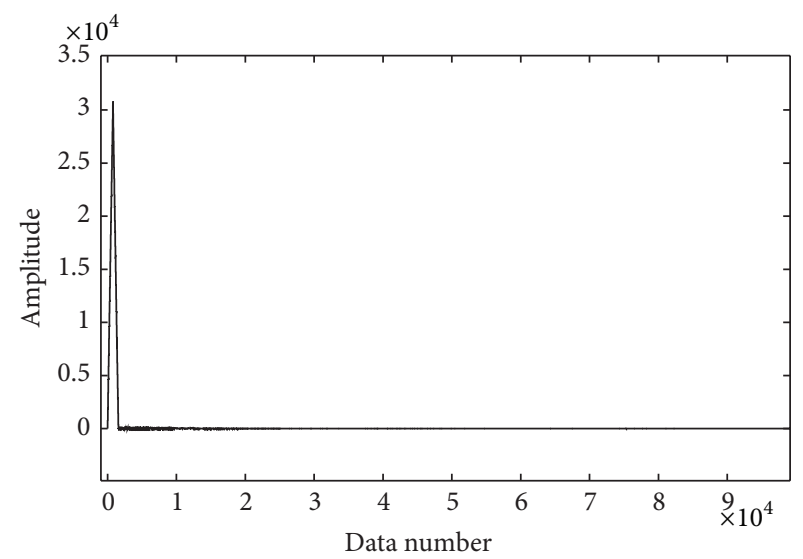

(b)

FIGURE 11: Wavelet coefficients of the vibration signal induced by outer race fault: (a) without PPR preprocessing and (b) with PPR preprocessing.

which we can see that, after employing peak-based piecewise recombination (PPR) preprocessing, energies of the signal are concentrated into only a few coefficients in wavelet domain, $99.9 \%$ in low frequency bands, compared with $49.31 \%$ without peak-based piecewise recombination.

The experimental results of inner race fault and roller element fault are similar to those of the outer race fault, which are shown in Figures 12 and 13.

\subsection{Comparisons between Traditional Wavelet-Based Detec-} tion Method and the Proposed Strategy with Envelop Demodulation. Envelop demodulation is always combined with wavelet-based denoising and enhancement methods to analyze vibration signals. Comparisons of the results after envelop demodulation based on the results of Section 4.3 are presented in this section.

Envelop demodulation is applied to the signals reconstructed from the thresholding of wavelet coefficients, and a backward peak-based piecewise recombination is implemented for the proposed strategy. The demodulated envelope spectra are shown in Figures 14-16. In Figure 14(a), although the fault frequency can be figured out, the magnitude of 


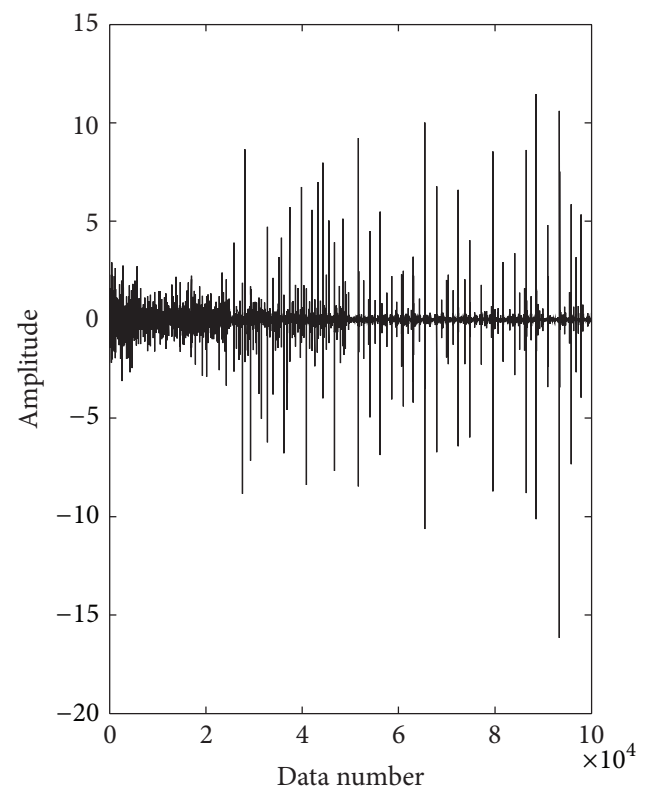

(a)

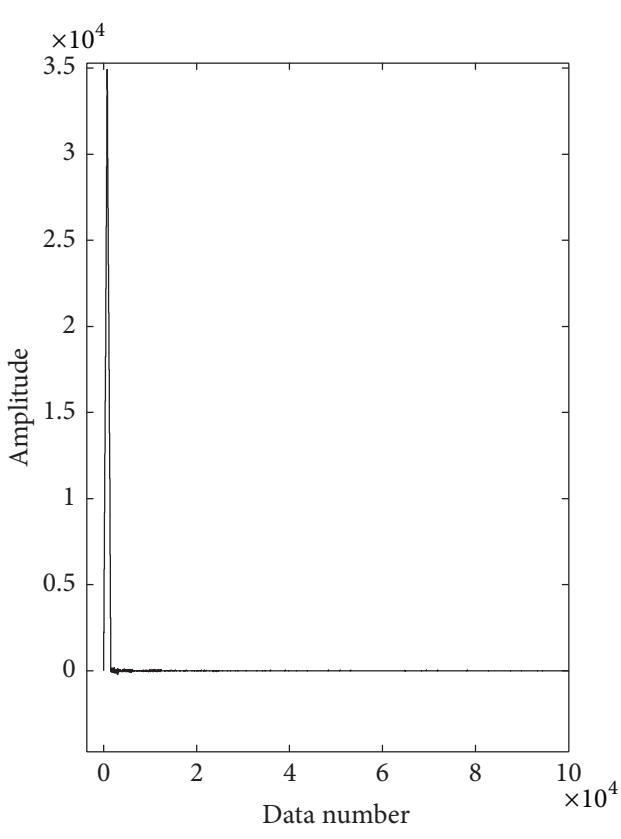

(b)

FIGURE 12: Wavelet coefficients of the vibration signal induced by inner race fault: (a) without PPR preprocessing and (b) with PPR preprocessing.

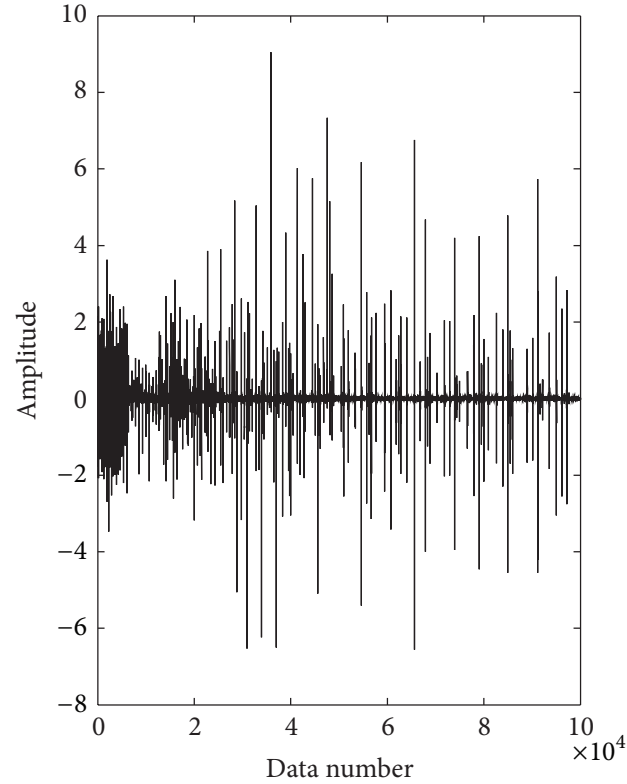

(a)

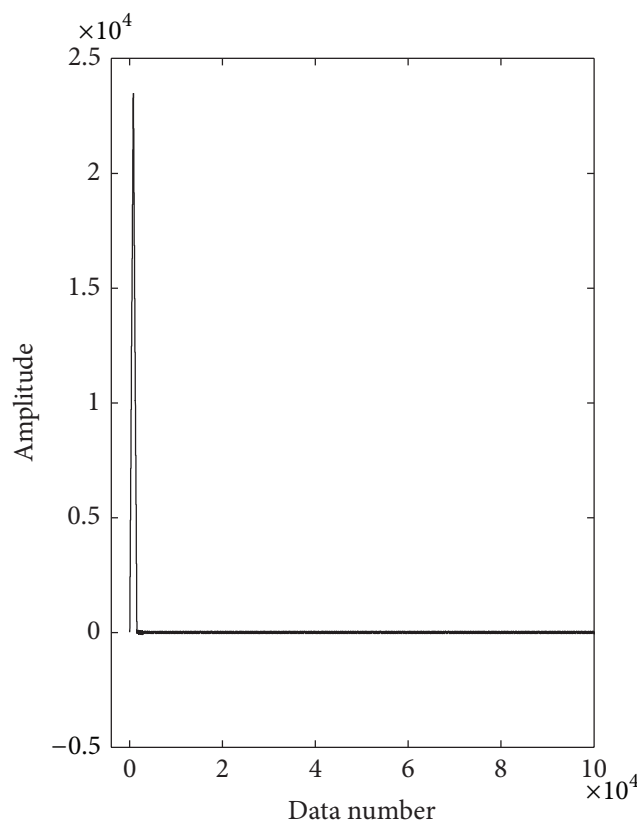

(b)

FIGURE 13: Wavelet coefficients of the vibration signal induced by roller element fault: (a) without PPR preprocessing and (b) with PPR preprocessing.

the outer race defect frequency is not the most remarkable one among all frequency components; thus, it is not always certain that a bearing defect exists in outer race. With the proposed method presented in this paper, the magnitude of outer race defect frequency becomes more prominent, where the magnitudes of outer race defect frequency have been enhanced and are more easily detected, as Figure 14(b) depicted. The proposed strategy is also more effective for inner race and roller bearing fault detection of rolling bearings, and the results are shown as in Figures 15 and 16. 


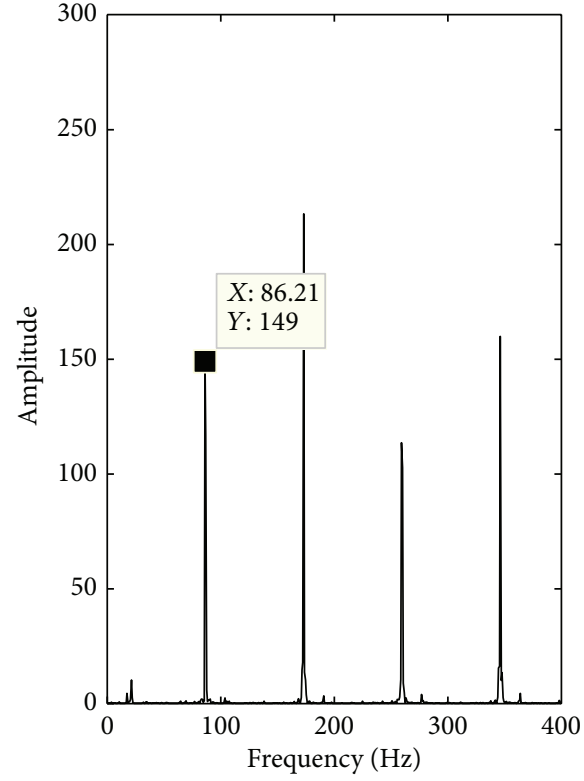

(a)

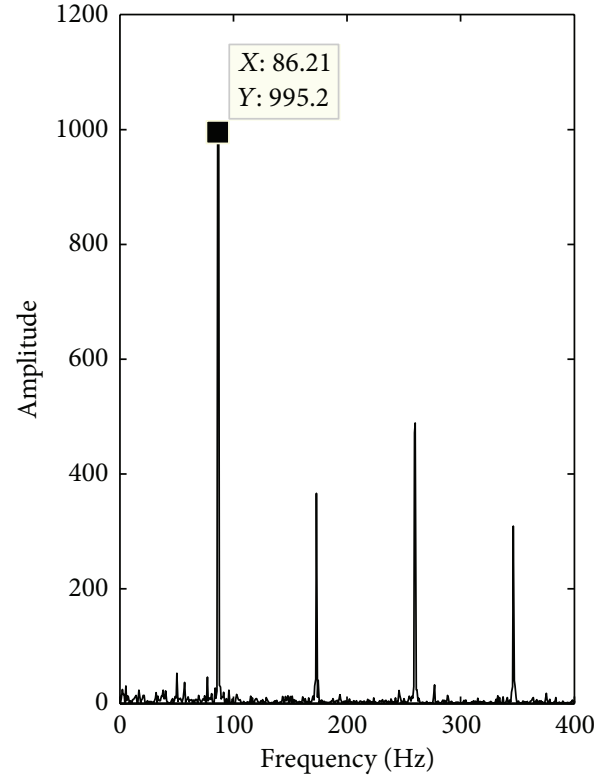

(b)

FIGURE 14: Envelope spectra of the vibration signal induced by outer race fault: (a) wavelet analysis method and (b) the proposed method.

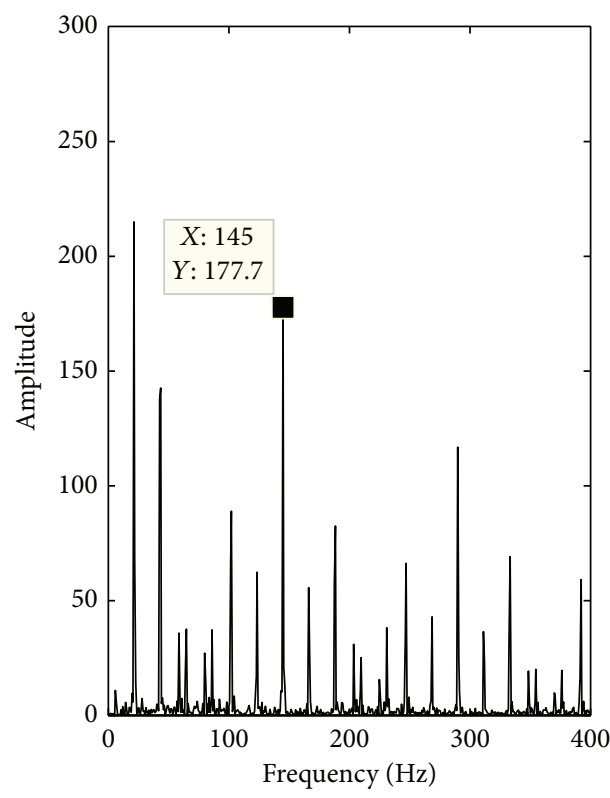

(a)

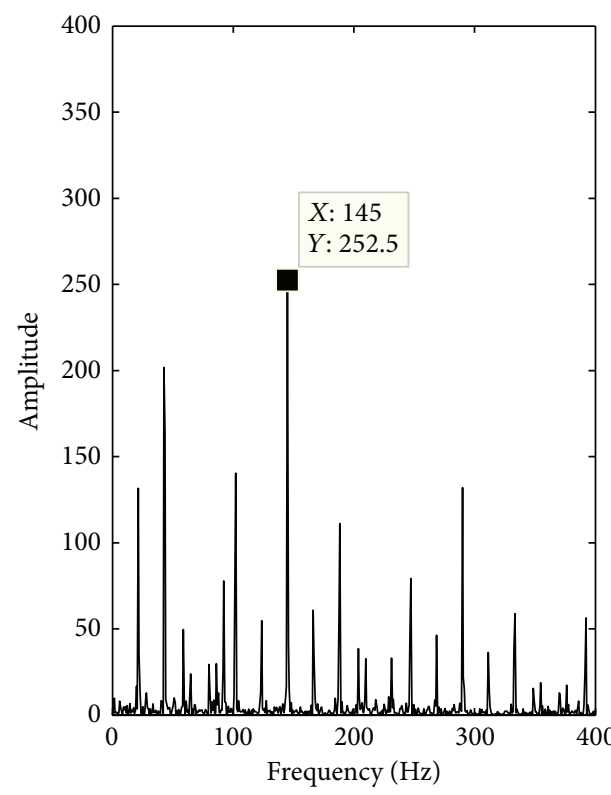

(b)

FIGURE 15: Envelope spectra of the vibration signal induced by inner race fault: (a) wavelet analysis method and (b) the proposed method.

If defect exists in the bearing, the magnitudes of faults frequencies will increase in the envelope spectrum. Therefore, they are often used to describe the fault severity and also employed to judge whether the fault features are enhanced. In this work, magnitudes of faults frequencies are chosen as the major indictors to reflect the fault features. As recorded and listed in Table 2, with the proposed method, magnitudes of faults frequencies are all enhanced and they become more easily distinguished from interfered noise for the experimental faults.

\section{Conclusions}

In order to improve the current wavelet-based method for fault detection of rolling bearings, the present paper developed a new way to improve vibration signals' smoothness 


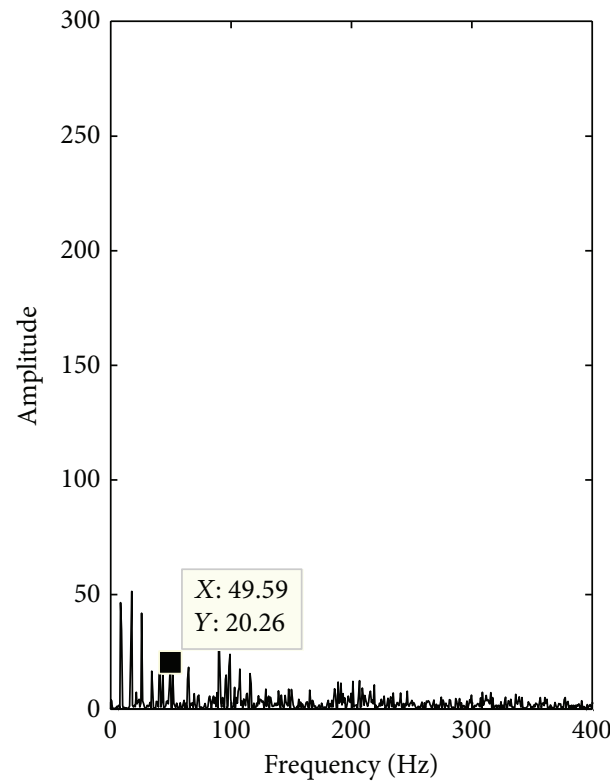

(a)

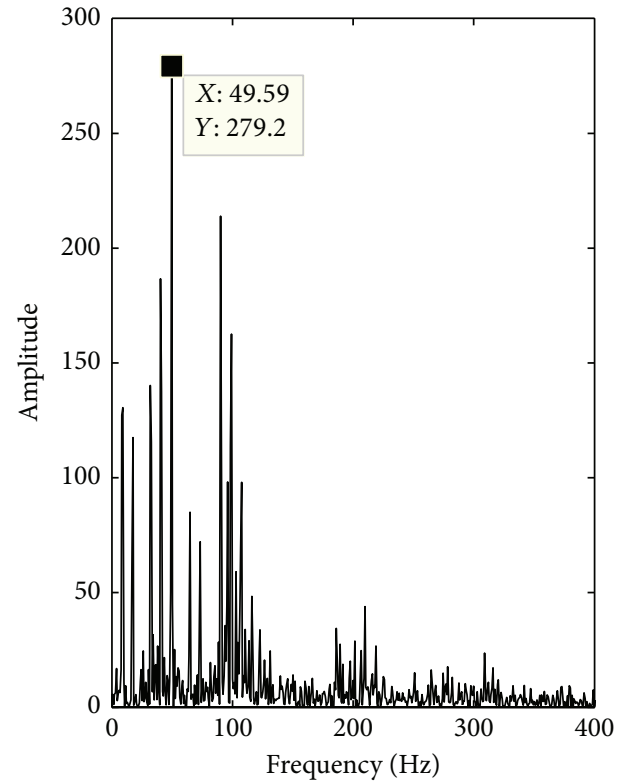

(b)

FIGURE 16: Envelope spectra of the vibration signal induced by roller element fault: (a) wavelet analysis method and (b) the proposed method.

TABLE 2: Major indictors for bearing fault features.

\begin{tabular}{lcc}
\hline $\begin{array}{l}\text { Defect frequency } \\
\text { magnitude of }\end{array}$ & $\begin{array}{c}\text { Traditional } \\
\text { wavelet-based } \\
\text { method }\end{array}$ & The proposed method \\
\hline outer race & 149 & 995.2 \\
inner race & 177.7 & 252.5 \\
roller element & 20.26 & 279.2 \\
\hline
\end{tabular}

as well as their sparseness in wavelet domain by employing peak-based piecewise recombination and envelope demodulation. Through the proposed method, most noise mixed in vibration signals can be eliminated and the weak fault signals of rolling bearings can be enhanced and become more easily detectable. Therationality and validity of the methods are also proved by various experiments. It shows that, compared with traditional wavelet-based method, the proposed method has many advantages in bearing fault signals enhancement and detection and is very simple and easy to implement.

There is still room to improve the present work. The sensitivity of the method to noise and methods to make energy more concentrated are what will be studied in future work.

\section{Conflict of Interests}

The authors declare that there is no conflict of interests regarding the publication of this paper.

\section{Acknowledgments}

This project is partially supported by the National Natural Science Foundation of China (Grant no. 51375037) and the National Program on Key Basic Research Project (Grant no. 2012CB026000). The third author would also like to thank the support of China Fundamental Research Funds for the Central Universities (ZY1410) and the Public Hatching Platform for Recruited Talents of Beijing University of Chemical Technology.

\section{References}

[1] W. Zhou, T. G. Habetler, and R. G. Harley, "Bearing condition monitoring methods for electric machines: a general review," in Proceedings of the IEEE International Symposium on Diagnostics for Electric Machines, Power Electronics and Drives (SDEMPED '07), pp. 3-6, September 2007.

[2] H. Q. Wang and P. Chen, "Intelligent diagnosis method for a centrifugal pump using features of vibration signals," Neural Computing and Applications, vol. 18, no. 4, pp. 397-405, 2009.

[3] H. Wang and P. Chen, "Sequential diagnosis for rolling bearing using fuzzy neural network," in Proceedings of the IEEE/ASME International Conference on Advanced Intelligent Mechatronics (AIM '08), pp. 56-61, Xi'an, China, August 2008.

[4] T. Mitoma, H. Wang, and P. Chen, "Fault diagnosis and condition surveillance for plant rotating machinery using partiallylinearized neural network," Computers and Industrial Engineering, vol. 55, no. 4, pp. 783-794, 2008.

[5] P. D. McFadden and J. D. Smith, "Vibration monitoring of rolling element bearings by the high-frequency resonance technique-a review," Tribology International, vol. 17, no. 1, pp. 3-10, 1984. 
[6] N. Tandon and A. Choudhury, "Review of vibration and acoustic measurement methods for the detection of defects in rolling element bearings," Tribology International, vol. 32, no. 8, pp. 469-480, 1999.

[7] R. B. W. Heng and M. J. M. Nor, "Statistical analysis of sound and vibration signals for monitoring rolling element bearing condition," Applied Acoustics, vol. 53, no. 1-3, pp. 211-226, 1998.

[8] C. Junsheng, Y. Dejie, and Y. Yu, "The application of energy operator demodulation approach based on EMD in machinery fault diagnosis," Mechanical Systems and Signal Processing, vol. 21, no. 2, pp. 668-677, 2007.

[9] R. B. Randall, J. Antoni, and S. Chobsaard, "The relationship between spectral correlation and envelope analysis in the diagnostics of bearing faults and other cyclostationary machine signals," Mechanical Systems and Signal Processing, vol. 15, no. 5, pp. 945962, 2001.

[10] A. B. Ming, Z. Y. Qin, W. Zhang et al., "Spectrum auto-correlation analysis and its application to fault diagnosis of rolling element bearings," Mechanical Systems and Signal Processing, vol. 41, no. 1, pp. 141-154, 2013.

[11] D. Yu, J. Cheng, and Y. Yang, "Application of EMD method and Hilbert spectrum to the fault diagnosis of roller bearings," Mechanical Systems and Signal Processing, vol. 19, no. 2, pp. 259270, 2005.

[12] V. K. Rai and A. R. Mohanty, "Bearing fault diagnosis using FFT of intrinsic mode functions in Hilbert-Huang transform," Mechanical Systems and Signal Processing, vol. 21, no. 6, pp. 26072615, 2007.

[13] Y. Lei, N. Li, J. Lin, and S. Wang, "Fault diagnosis of rotating machinery based on an adaptive ensemble empirical mode decomposition," Sensors, vol. 13, no. 12, pp. 16950-16964, 2013.

[14] J. Cheng, Y. Yang, and Y. Yang, "A rotating machinery fault diagnosis method based on local mean decomposition," Digital Signal Processing, vol. 22, no. 2, pp. 356-366, 2012.

[15] B. Chen, Z. He, X. Chen, H. Cao, G. Cai, and Y. Zi, "A demodulating approach based on local mean decomposition and its applications in mechanical fault diagnosis," Measurement Science and Technology, vol. 22, no. 5, Article ID 055704, 2011.

[16] S. Prabhakar, A. R. Mohanty, and A. S. Sekhar, "Application of discrete wavelet transform for detection of ball bearing race faults," Tribology International, vol. 35, no. 12, pp. 793-800, 2002.

[17] R. Yan, R. X. Gao, and X. Chen, "Wavelets for fault diagnosis of rotary machines: a review with applications," Signal Processing, vol. 96, part A, pp. 1-15, 2014.

[18] H. Wang and P. Chen, "A feature extraction method based on information theory for fault diagnosis of reciprocating machinery," Sensors, vol. 9, no. 4, pp. 2415-2436, 2009.

[19] K. Li, X. L. Ping, H. Q. Wang, and P. Chen, "Sequential fuzzy diagnosis method for motor roller bearing in variable operating conditions based on vibration analysis," Sensors, vol. 13, no. 6, pp. 8013-8041, 2013.

[20] R. Rubini and U. Meneghetti, "Application of the envelope and wavelet transform analyses for the diagnosis of incipient faults in ball bearings," Mechanical Systems and Signal Processing, vol. 15, no. 2, pp. 287-302, 2001.

[21] L. Gao, Z. Yang, L. Cai, H. Wang, and P. Chen, "Roller bearing fault diagnosis based on nonlinear redundant lifting wavelet packet analysis," Sensors, vol. 11, no. 1, pp. 260-277, 2011.

[22] Z. Yang, L. Cai, L. Gao, and H. Wang, "Adaptive redundant lifting wavelet transform based on fitting for fault feature extraction of roller bearings," Sensors, vol. 12, no. 4, pp. 4381-4398, 2012.
[23] Z. He, "Peak transform for efficient image representation and coding," IEEE Transactions on Image Processing, vol. 16, no. 7, pp. 1741-1754, 2007.

[24] S. Anila and N. Devarajan, "The usage of peak transform for image compression," International Journal of Computer Systems Science \& Engineering, vol. 2, no. 11, pp. 6308-6316, 2010.

[25] D. Ho and R. B. Randall, "Optimization of bearing diagnostic techniques using simulated and actual bearing fault signals," Mechanical Systems and Signal Processing, vol. 14, no. 5, pp. 763$788,2000$. 


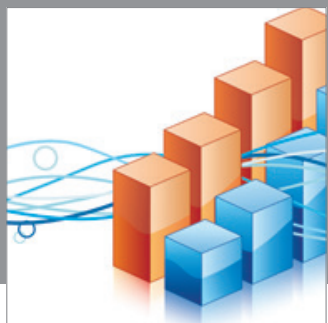

Advances in

Operations Research

mansans

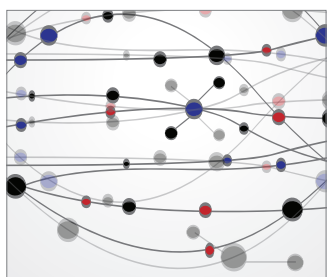

The Scientific World Journal
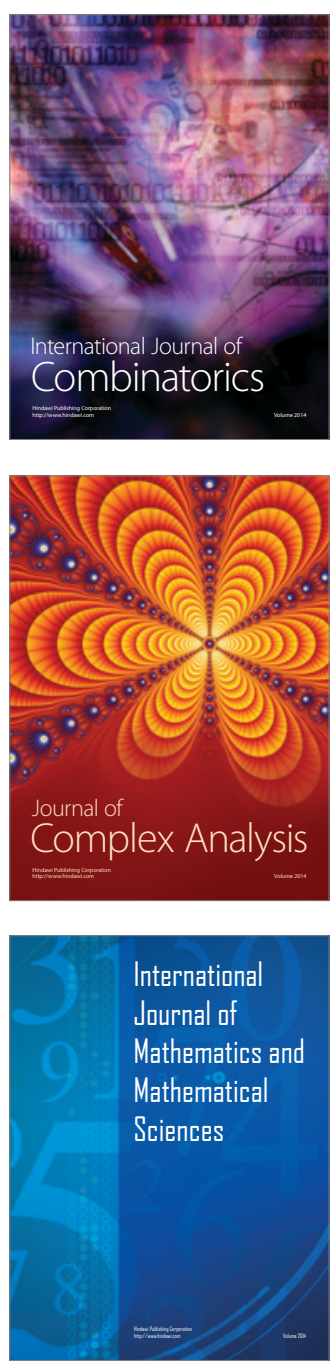
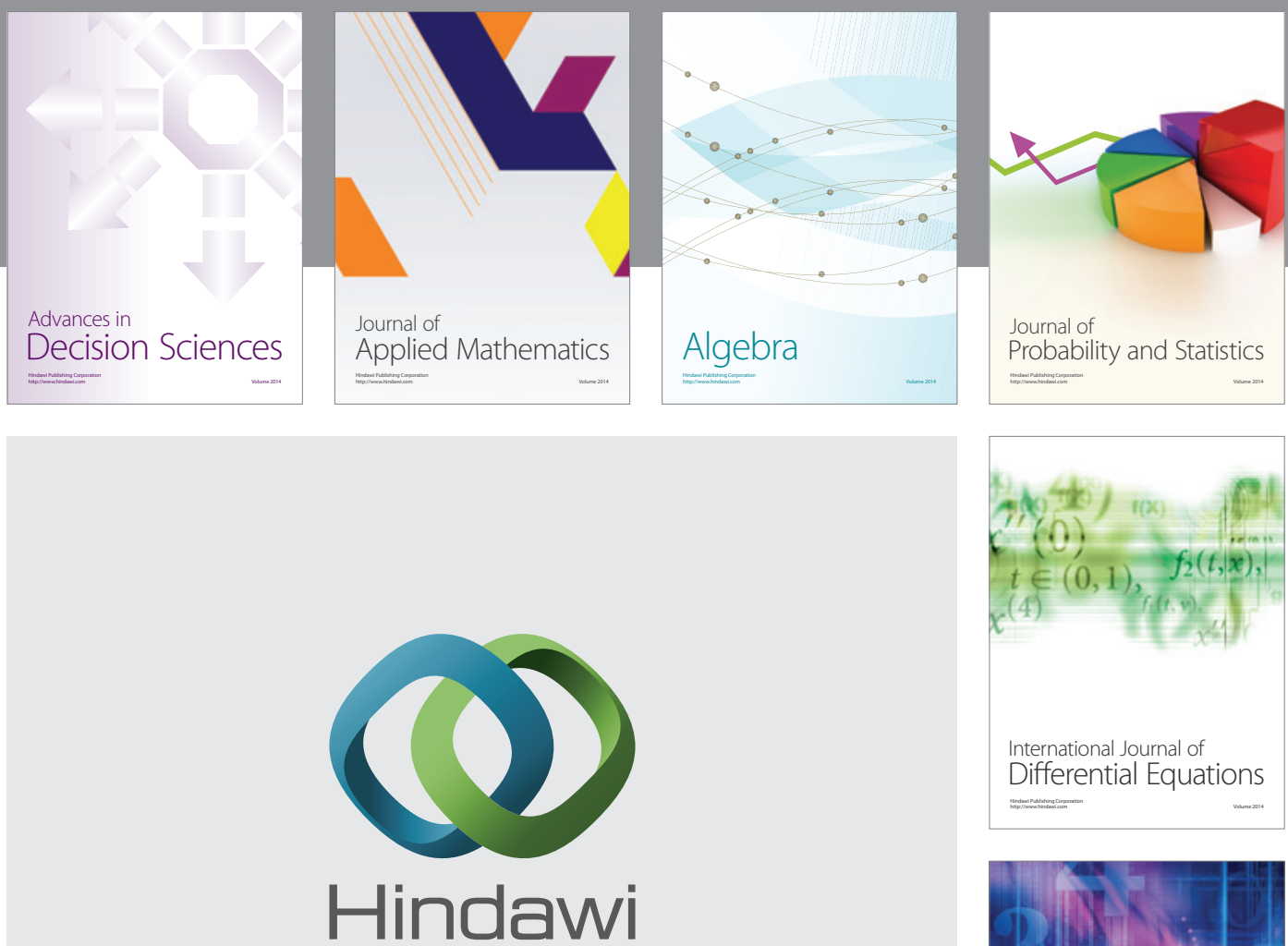

Submit your manuscripts at http://www.hindawi.com
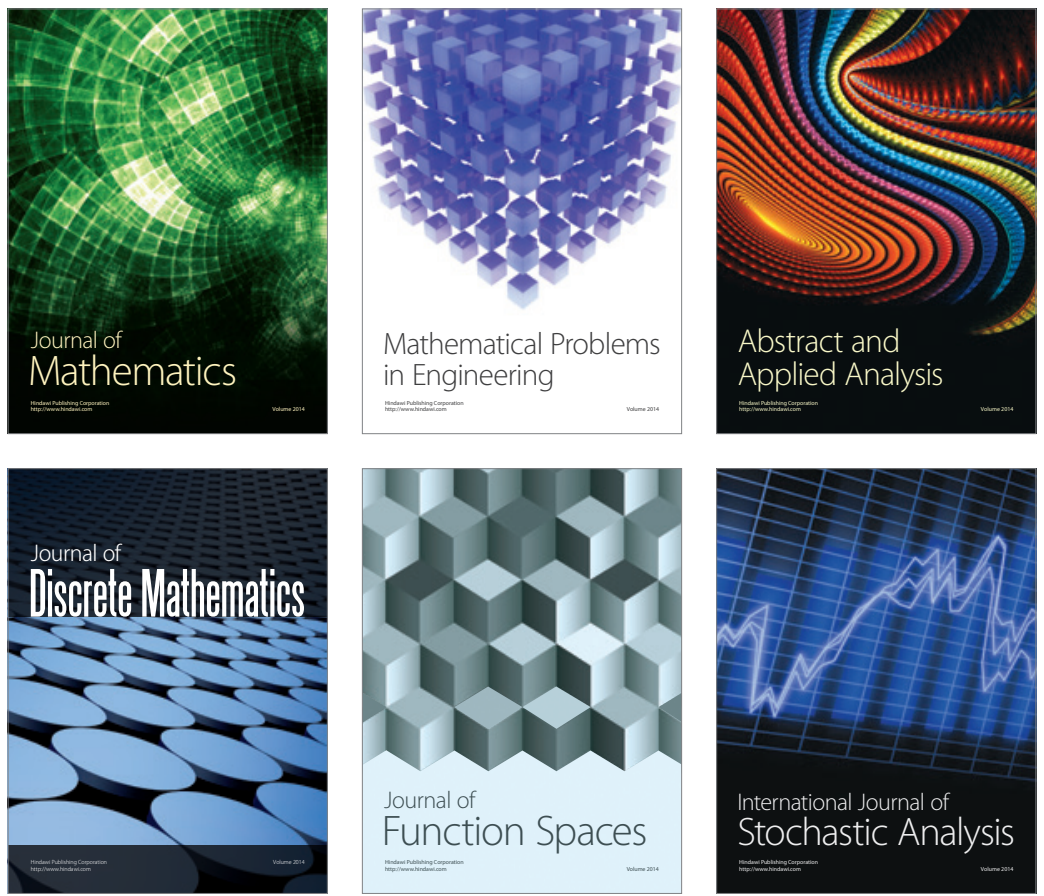

Journal of

Function Spaces

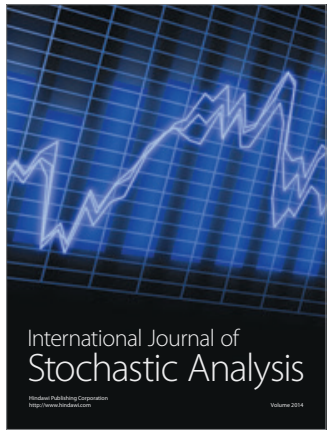

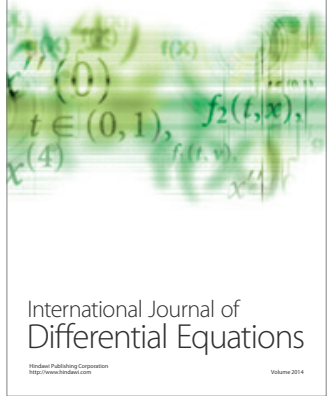
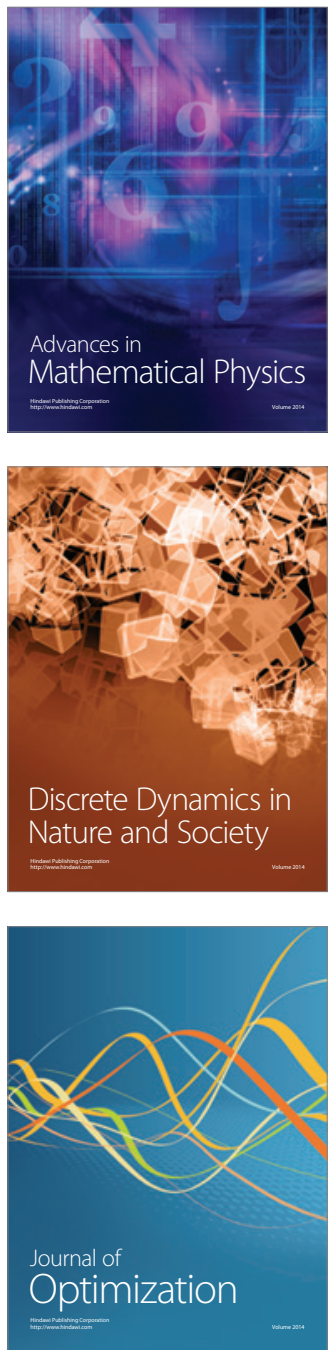